ARTICLE

DOI: $10.1038 / s 41467-018-04483-3$

\title{
Observation of different reactivities of para and ortho-water towards trapped diazenylium ions
}

Ardita Kilaj ${ }^{1}$, Hong Gao ${ }^{1,6}$, Daniel Rösch¹, Uxia Rivero ${ }^{1}$, Jochen Küpper (i) ${ }^{2,3,4,5}$ \& Stefan Willitsch ${ }^{1}$

Water is one of the most fundamental molecules in chemistry, biology and astrophysics. It exists as two distinct nuclear-spin isomers, para- and ortho-water, which do not interconvert in isolated molecules. The experimental challenges in preparing pure samples of the two isomers have thus far precluded a characterization of their individual chemical behavior. Capitalizing on recent advances in the electrostatic deflection of polar molecules, we separate the ground states of para- and ortho-water in a molecular beam to show that the two isomers exhibit different reactivities in a prototypical reaction with trapped diazenylium ions. Based on $a b$ initio calculations and a modelling of the reaction kinetics using rotationally adiabatic capture theory, we rationalize this finding in terms of different rotational averaging of iondipole interactions during the reaction.

\footnotetext{
${ }^{1}$ Department of Chemistry, University of Basel, Klingelbergstrasse 80, Basel 4056, Switzerland. ${ }^{2}$ Center for Free-Electron Laser Science, Deutsches Elektronen-Synchrotron DESY, Notkestrasse 85, Hamburg 22607, Germany. ${ }^{3}$ Department of Physics, Universität Hamburg, Luruper Chaussee 149, Hamburg 22761, Germany. ${ }^{4}$ Department of Chemistry, Universität Hamburg, Martin-Luther-King-Platz 6, Hamburg 20146, Germany. ${ }^{5}$ The Hamburg Center for Ultrafast Imaging, Universität Hamburg, Luruper Chaussee 149, Hamburg 22761, Germany. ${ }^{6}$ Present address: Beijing National Laboratory of Molecular Sciences, State Key Laboratory of Molecular Reaction Dynamics, Institute of Chemistry, Chinese Academy of Sciences, Beijing 100190, China. These authors contributed equally: Ardita Kilaj, Hong Gao. Correspondence and requests for materials should be addressed to S.W. (email: stefan.willitsch@unibas.ch)
} 
W ater, $\mathrm{H}_{2} \mathrm{O}$, is one of the key molecules in nature, it acts as the fundamental solvent in biological systems and is one of the major molecular constituents of the universe. It exists in two forms, para(p)-water and ortho(o)-water, which are distinguished by their values of the quantum number of the total nuclear spin $I$, where $I=0$ and 1 for $p$ - and $o-\mathrm{H}_{2} \mathrm{O}$, respectively. Interconversion of the nuclear-spin isomers is forbidden in isolated molecules and nuclear-spin symmetry is usually conserved in collisions, by electromagnetic radiation and even in chemical reactions ${ }^{1-3}$. However, nuclear-spin-symmetry interconversion has been observed in a variety of polyatomic molecules and has been rationalized to occur via doorway states with a mixed nuclear-spin character ${ }^{2,4,5}$. For water in the vapor and condensed phases, the para/ortho-interconversion rates reported in the literature vary widely and remain a controversial topic $^{6-8}$.

Apart from their total nuclear spin, para- and ortho-water also differ in other important respects. Because the generalized Pauli principle dictates that the total molecular wavefunction has to be antisymmetric under the permutation of the two hydrogen nuclei in the molecule ${ }^{1}$, (ortho-) para-water is associated with (anti) symmetric rotational functions in the electronic-vibrational ground state of the molecule. Thus, nuclear-spin and rotational symmetry are intimately linked ${ }^{9}$. As a consequence, the ground state of para-water is the absolute rotational ground state $\left|j_{K_{a} K_{c}}\right\rangle=\left|0_{00}\right\rangle$, whereas the ground state of ortho-water is the first excited rotational state $\left|j_{K_{a} K_{c}}\right\rangle=\left|1_{01}\right\rangle$. Here, $j$ denotes the quantum number of the rotational angular momentum and $K_{a}$ and $K_{c}$ are the quantum numbers of the projection of the rotational angular momentum on the $a$ and $c$ principal axes of inertia of the molecule, respectively.

Considering the different properties of its two nuclear-spin isomers and the eminent importance of water in a variety of chemical contexts, it begs the question whether para- and orthowater also show different chemical behavior. In a wider context, this problem ties into ongoing efforts to understand how different molecular degrees of freedom (translation, nuclear spin, rotation, vibration, electronic motion) and the interplay between them influence chemical reactivity. Despite the significant amount of studies focusing on vibrational effects in chemical reactions $s^{10-12}$, the roles of nuclear spin and molecular rotation have scarcely been explored experimentally. This is mainly due to the fact that rotational energy transfer is likely to happen in any collision rendering it difficult to prepare molecules in specific rotational levels ${ }^{13,14}$ so that only comparatively few rotational-state resolved studies have been reported so far ${ }^{15-17}$. A similar scarcity of data exists with regard to studies involving individual nuclear-spin isomers ${ }^{18}$ which are in general difficult to separate and to prepare individually 9,19 .

Recent progress in manipulating polar molecules using electrostatic fields has made it possible to select and spatially separate different conformers and rotational states of molecules in supersonic molecular beams ${ }^{14}$. By combining this technology with a stationary reaction target of Coulomb-crystallized ions in a linear-quadrupole ion trap (LQT) ${ }^{20,21}$, we have recently studied conformer-selected molecule-ion reaction dynamics and observed that reaction-rate constants can strongly depend on molecular conformation ${ }^{22,23}$. Here, we extend this method to the separation of different nuclear-spin isomers using their different rotational properties in order to study ion-molecule reactions with control over the rotational and nuclear-spin state of the neutral reaction partner, a field on which only scarce experimental data are available so far. Our approach is particularly suited for polyatomic neutrals for which rigorous rotational state preparation is difficult to achieve solely by supersonic cooling ${ }^{16}$. As an example, we investigate the proton-transfer reaction of water with ionic diazenylium $\left(\mathrm{N}_{2} \mathrm{H}^{+}\right)$,

$$
\mathrm{H}_{2} \mathrm{O}+\mathrm{N}_{2} \mathrm{H}^{+} \rightarrow \mathrm{N}_{2}+\mathrm{H}_{3} \mathrm{O}^{+},
$$

an important molecule in astrochemistry which has been observed in the interstellar medium ${ }^{24}$. Its detection has proven crucial to trace molecular nitrogen in pre-stellar clouds to understand the early stages of star formation 25,26 .

We find that the para-species reacts $23(9) \%$ faster than the ortho-isomer. Based on ab initio calculations and a modelling of the reaction kinetics using rotationally adiabatic capture theory, we rationalize this finding in terms of different rotational averaging of ion-dipole interactions during the reaction.

\section{Results}

Experimental approach. The two ground states of para- and ortho-water show distinct responses to an electric field, i.e., different Stark-energy shifts and correspondingly different effective space-fixed dipole moments (Supplementary Note 1 and $^{9}$ ). This enables their spatial separation by the electric field gradient of an electrostatic deflector ${ }^{9,14}$.

The experimental setup is schematically depicted in Fig. 1. It consists of a molecular-beam machine equipped with the electrostatic deflector connected to an ultrahigh-vacuum chamber housing an ion trap ${ }^{23,27}$. A beam of internally cold molecules was formed in a pulsed supersonic expansion of water seeded in argon carrier gas (Methods). The molecular beam was collimated by two skimmers before entering the electrostatic deflector. A voltage of $15 \mathrm{kV}$ was applied across the deflector electrodes in order to generate a vertical electric field gradient for the spatial separation of the two nuclear-spin isomers. After passing another skimmer, the beam was directed towards a LQT. The trap was loaded with Coulomb crystals of laser-cooled $\mathrm{Ca}^{+}$ions ${ }^{20,22}$ as well as sympathetically cooled $\mathrm{N}_{2} \mathrm{H}^{+}$reactant ions (image inset in Fig. 1). By vertically tilting the molecular beam apparatus relative to the LQT, different regions of the deflected molecular beam were overlapped with the Coulomb crystals ${ }^{23}$. The tilting angle of the molecular beam apparatus defined a deflection coordinate $y$ for molecules arriving at the trap center. After exposure to the molecular beam for a variable time period, the Coulomb crystals were ejected into a high-resolution time-of-flight mass spectrometer (TOF-MS) ${ }^{27}$ for the mass and quantitative analysis of their constituents.

Isomer populations in the deflected water beam. In order to probe its composition in terms of quantum states and to characterize the spatial separation of the two nuclear-spin isomers, density profiles of the molecular beam were measured. A pulsed ultraviolet laser beam was used to generate water ions by $(2+1)$ resonance-enhanced multi-photon ionization (REMPI) via selected rotational levels of the $\tilde{C}$ electronic state ${ }^{28}$. The ions were subsequently ejected into the TOF-MS. This technique enabled the selective detection of the ground states of either para- or ortho-water and the determination of the individual density profiles of the two isomers in the beam. From a REMPI spectrum of an undeflected water beam, it was confirmed that the supersonic expansion was composed predominantly of the $j_{K_{a} K_{c}}=0_{00}$ and $1_{01}$ rotational states of $\mathrm{H}_{2} \mathrm{O}$, i.e., the ground states of $p$ - and $o-\mathrm{H}_{2} \mathrm{O}$, respectively. A possible minor contribution from the $1_{10}$ state did not interfere with the present experiments (Supplementary Note 2 and 3 ).

Figure 2a shows deflection profiles of para (red diamonds) and ortho- (blue squares) water obtained from the ion signal at massto-charge ratio $\mathrm{m} / \mathrm{z}=18 \mathrm{u}$ recorded as a function of the deflection coordinate $y$ at a deflector voltage of $15 \mathrm{kV}$. The purple circles represent the sum of the para and ortho-profiles. 


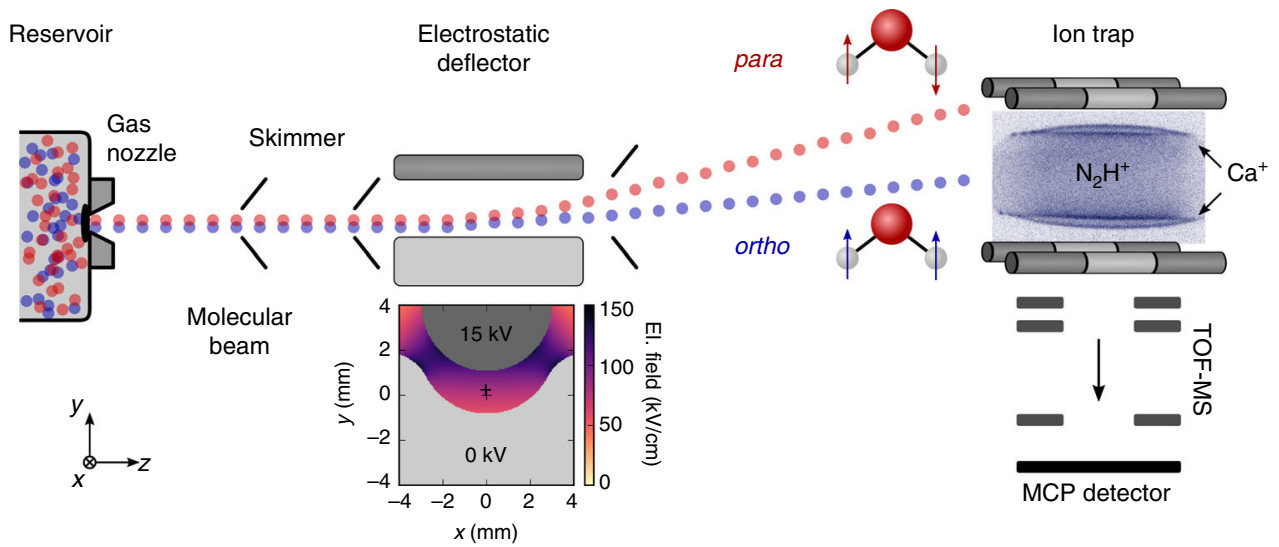

Fig. 1 Schematic of the experimental setup. A pulsed molecular beam of water molecules seeded in argon emanates from a room-temperature reservoir through a pulsed gas nozzle and passes an electrostatic deflector. The inhomogeneous electric field inside the deflector (shown in the inset below) spatially separates para- and ortho-water molecules due to their different effective dipole moments. After the deflector, the beam is directed at an ion trap containing a Coulomb crystal of $\mathrm{Ca}^{+}$and sympathetically cooled $\mathrm{N}_{2} \mathrm{H}^{+}$reactant ions (inset image). The products and kinetics of reactive collisions between $\mathrm{N}_{2} \mathrm{H}^{+}$and $\mathrm{H}_{2} \mathrm{O}$ are probed using a time-of-flight mass spectrometer (TOF-MS) ${ }^{27}$

a

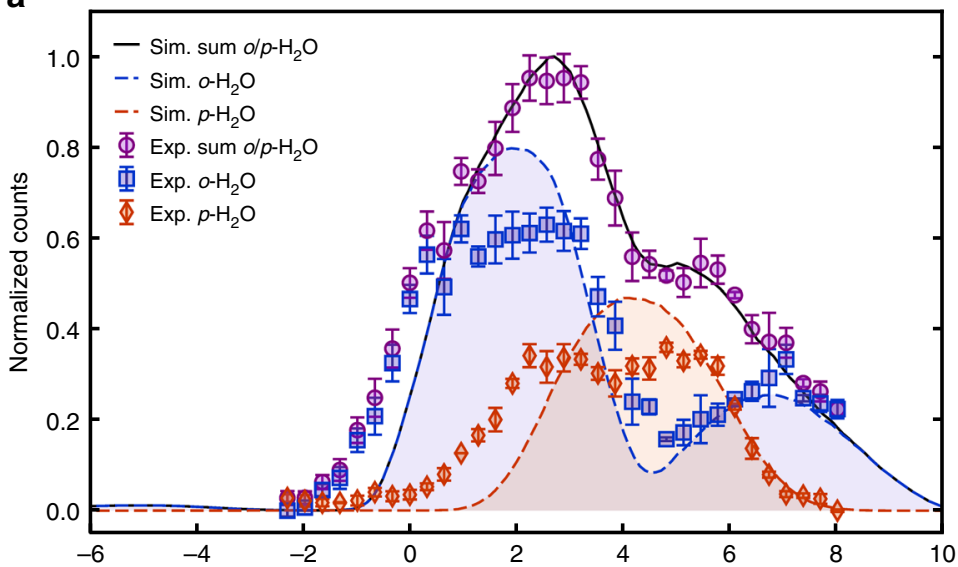

b

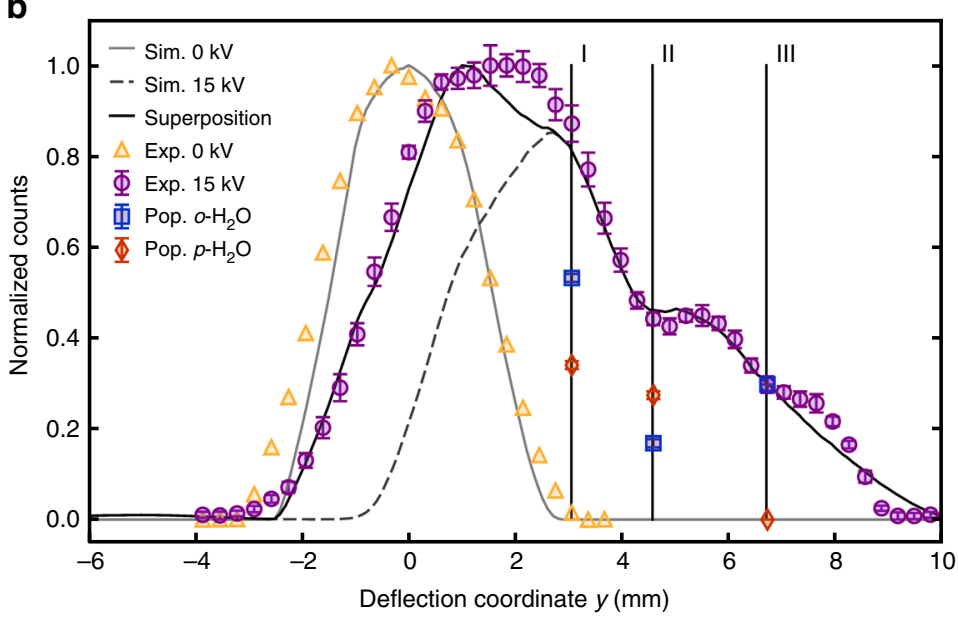

C
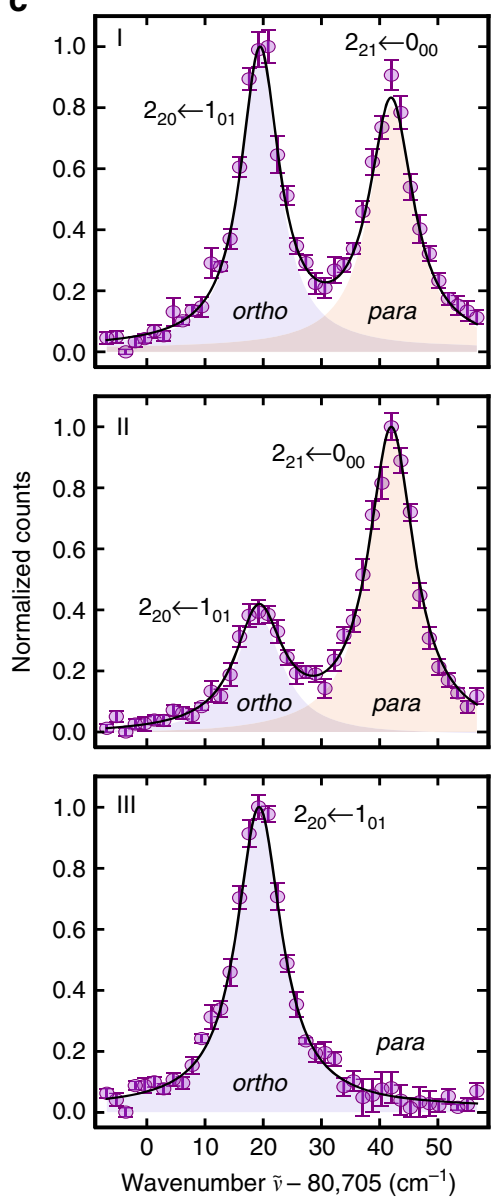

Fig. 2 Molecular-beam deflection profiles of the ground states of para and ortho-water. a Experimental isomer-specific density profiles of $o$ - (blue squares) and $p-\mathrm{H}_{2} \mathrm{O}$ (red diamonds) in the deflected molecular beam (deflector voltage $15 \mathrm{kV}$ ) measured by $(2+1) \mathrm{REMPI}$ together with the total deflection profile (sum of the ortho- and para-signals, purple circles). The lines represent Monte-Carlo trajectory simulations of the deflection profiles. The contributions from ortho- and para-water are indicated by the blue and red shaded areas, respectively. $\mathbf{b}$ Total water deflection profile measured by femtosecond-laser ionization for deflector voltages of $0 \mathrm{kV}$ (yellow triangles) and $15 \mathrm{kV}$ (purple circles). The three vertical lines marked I, II, and III indicate the deflection coordinates at which reaction rates were measured. The red/blue symbols represent the relative populations of the isomers normalized to the total signal at positions I, II, and III as determined from the REMPI spectra shown in c. c REMPI spectra of $\mathrm{H}_{2} \mathrm{O}$ measured at the three positions I, II, III (purple circles). The two peaks observed at $80,724 \mathrm{~cm}^{-1}$ and $80,747 \mathrm{~cm}^{-1}$ correspond to transitions from the ground states of ortho- and para-water, respectively. The peaks are fitted with a sum of two Lorentzians (solid black line) with contributions from ortho- and para-isomers depicted as blue and red shaded areas, respectively. Error bars correspond to one standard error of at least three independent measurements 


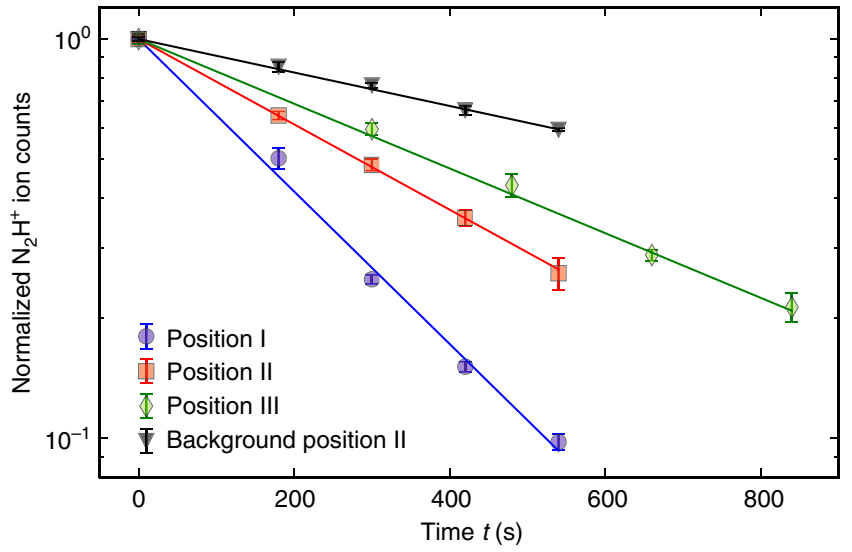

Fig. 3 Reaction-rate measurements at the deflection coordinates I-III indicated in Fig. 2b. The data are normalized to the ion signal at time $t=0$. The lines represent fits to the data according to an integrated pseudo-firstorder rate law. The black triangles show an example of a measurement of the reaction rate with background gas at position II for comparison. Error bars correspond to one standard error of four independent measurements

The different projections of the angular momentum of the ground state of ortho-water onto the space-fixed direction of the electric field leads to two components, $M=0$ and $|M|=1$, which exhibit a weak and strong Stark shift, respectively. These correlate with the two peaks of the $o-\mathrm{H}_{2} \mathrm{O}$ deflection profile at low and high deflection coordinates, respectively. Contrarily, the para-isomer only has one angular-momentum projection component $M=0$ with an intermediate Stark shift such that its deflection profile shows a single peak situated in between the two peaks of the ortho-form. In this way, a partial spatial separation of the two isomers was achieved and the $o / p$-ratio was well defined at each deflection coordinate 9 . The solid and broken lines show corresponding theoretical deflection profiles derived from Monte Carlo trajectory simulations (Supplementary Note 1).

In addition, a femtosecond (fs) laser was employed to probe molecules reaching the trap center by strong-field ionization irrespective of the species or the internal quantum state ${ }^{29}$. Subsequent ion ejection into the TOF-MS enabled the determination of the combined relative density of para- and ortho-water molecules in the beam as a function of the deflection coordinate. The acquired beam profiles for deflector voltages of 0 and $15 \mathrm{kV}$ are presented in Fig. $2 \mathrm{~b}$ as yellow triangles and purple circles, respectively. Note that in these measurements, the gas valve was operated at a higher repetition rate $(200 \mathrm{~Hz})$ compared to the experiments shown in Fig. 2a $(20 \mathrm{~Hz})$ which leads to slightly different molecular-beam characteristics and therefore deflection profiles. At $15 \mathrm{kV}$, experiment and simulation (dashed grey line) agree well at large deflection coordinates, but differ significantly around $y=0$. In this region, the mass spectra indicate the presence of clusters formed in the supersonic expansion. A fs laser pulse can break these clusters resulting in water ions detected together with the water-monomer signal at $m / z=18 \mathrm{u}$ in the TOF-MS. Our data also show that these clusters are not deflected and do not contaminate the beam at deflection coordinates larger than $2 \mathrm{~mm}$ (Supplementary Note 3). This picture is corroborated by the reproduction of the experimental beam profile by a weighted superposition (black solid line) of simulations of the deflected water beam at $15 \mathrm{kV}$ and an undeflected beam at $0 \mathrm{kV}$ (grey solid line).

Probing the specific reactivities of the two isomers requires the preparation of samples with well defined para/ortho-ratios. Based on the deflection profiles and their simulations, three deflection coordinates with varying relative populations of para- and ortho- water were chosen. At each of these positions labelled I, II, and III in Fig. 2b, REMPI spectra were recorded from which the populations of para and ortho-water were determined (Fig. 2c). From fits of the intensities of the lines in the spectra, the relative populations of the two isomers were obtained (Supplementary Note 2). The populations of para (ortho-) water thus obtained were $39(1) \%(61(1) \%), 62(2) \%(38(2) \%)$ and $0 \%(100 \%)$ at positions I, II, and III. We note that at position III, the beam consists of pure ortho-water within the measurement uncertainties and therefore enables a direct measurement of the reaction rate constant of ortho-water.

Reaction-rate measurements. With the molecular beam prepared with well-known ratios of the two isomers at different deflection coordinates, measurements of the rate of reaction (1) were performed (Methods). First, a Coulomb crystal of about $1000 \mathrm{Ca}^{+}$ions was loaded into the LQT. Then, nitrogen gas was leaked into the vacuum chamber and $\mathrm{N}_{2}^{+}$ions were generated by fs-laser ionization. After the formation of $\mathrm{N}_{2}^{+}$ions in the trap, $\mathrm{H}_{2}$ gas was leaked into the vacuum chamber to quantitatively convert $\mathrm{N}_{2}^{+}$into $\mathrm{N}_{2} \mathrm{H}^{+}$via the reaction $\mathrm{N}_{2}^{+}+\mathrm{H}_{2} \rightarrow \mathrm{N}_{2} \mathrm{H}^{+}+\mathrm{H}$. The thus formed $\mathrm{N}_{2} \mathrm{H}^{+}$ions were sympathetically cooled into the Coulomb crystal and accumulated at its center (inset in Fig. 1). Subsequently, the molecular beam apparatus was set to a specific deflection coordinate and the deflector was turned on at a voltage of $15 \mathrm{kV}$ to direct the molecular beam at the Coulomb crystal and engage the reaction. After a variable period of exposure to the molecular beam, the reduction of the number of $\mathrm{N}_{2} \mathrm{H}^{+}$reactant ions was probed by ejecting the Coulomb crystal into the TOF$\mathrm{MS}^{27}$ (Fig. 3). In addition, the formation of $\mathrm{H}_{3} \mathrm{O}^{+}$as the ionic reaction product was verified using the TOF-MS. The rate measurements were repeated five times for each of the three deflection coordinates I-III. For every reaction measurement, a subsequent measurement of the rate of reaction of $\mathrm{N}_{2} \mathrm{H}^{+}$with the background gas in the vacuum chamber was performed by setting the deflector voltage to $0 \mathrm{kV}$ while keeping the deflection coordinate constant, thus pointing the molecular beam away from the center of the LQT. Since the number of water molecules is continuously replenished through the molecular beam, the rate constants could be determined within the framework of a pseudo-first-order kinetics treatment (Supplementary Note 4). The pseudo-firstorder rate constants of the reactions with background gas were directly subtracted from the total rate constants to give the rate constants for the reactions of the diazenylium ions with water from the molecular beam.

Isomer-specific rate constants. Combining the determinations of the total reaction-rate constants, of the relative populations of $o$ - and $p-\mathrm{H}_{2} \mathrm{O}$ at positions I, II, and III, and of the combined density profile of the deflected beam, the individual reaction-rate constants $k_{o}$ and $k_{p}$ of ortho- and para-water, respectively, were deduced. For each of the three deflection coordinates $y_{i}$ ( $i \in\{\mathrm{I}, \mathrm{II}, \mathrm{III}\})$, the total first-order rate constant $k_{\mathrm{tot}, i}^{\prime}$ is given by

$$
k_{\mathrm{tot}, i}^{\prime}=\tilde{n}_{i}\left(p_{o, i} k_{o}^{\prime}+p_{p, i} k_{p}^{\prime}\right)
$$

where $p_{o, i}$ and $p_{p, i}$ are the populations of ortho- and para-water, respectively, and $\tilde{n}_{i}$ are the relative densities of the water beam at positions $y_{i}$. $\tilde{n}_{i}$ is obtained from the beam profile $I(y)$ measured with the fs laser at $15 \mathrm{kV}$ (Fig. 2b) via $\tilde{n}_{i}=I\left(y_{i}\right) / I\left(y_{\mathrm{II}}\right)$ with position II taken as reference point. Once $k_{o}^{\prime}$ and $k_{p}^{\prime}$ were known, the relative difference of the reaction rates of the two isomers was calculated as $r=2\left(k_{p}^{\prime}-k_{o}^{\prime}\right) /\left(k_{p}^{\prime}+k_{o}^{\prime}\right)$.

The system of equations (2) was solved by a least-squares optimization. From the experimental data, the pseudo-first-order 
a

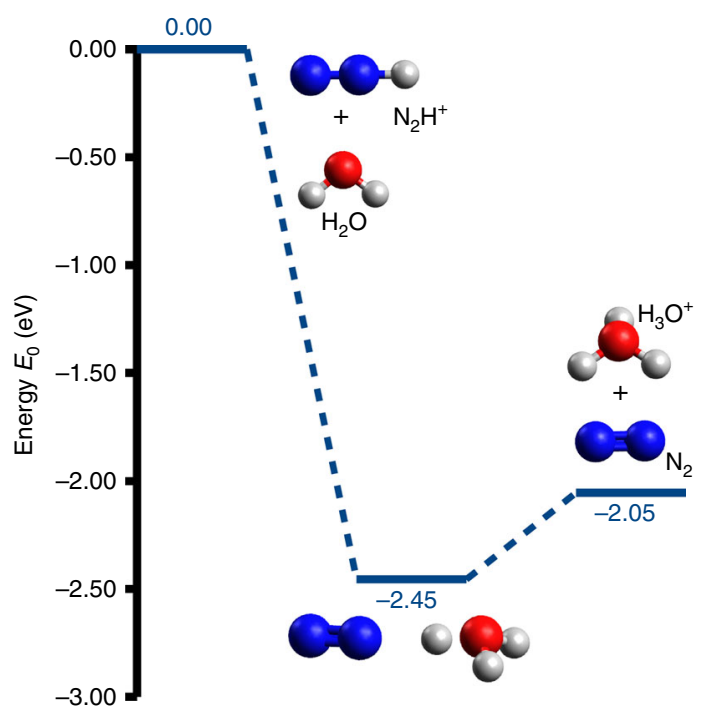

b

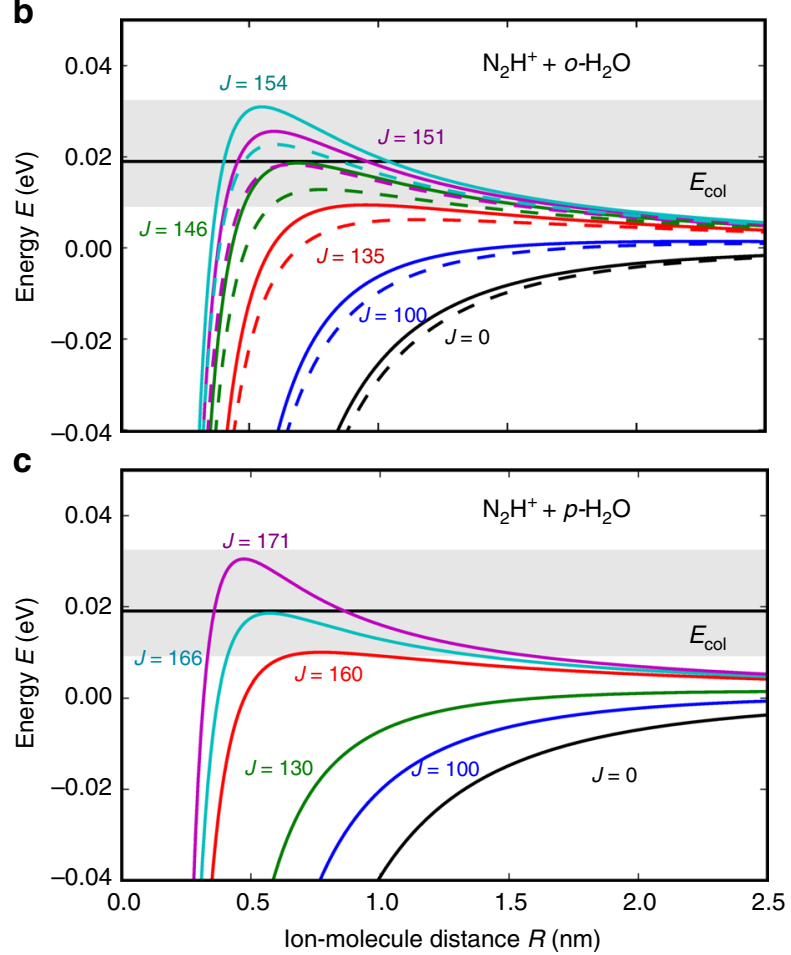

Fig. 4 Theoretical predictions from ab initio calculations and adiabatic capture theory. a Potential-energy profile along the reaction coordinate for the proton transfer reaction between $\mathrm{N}_{2} \mathrm{H}^{+}$and $\mathrm{H}_{2} \mathrm{O}$ at the CCSD/aug-cc-pVTZ level of theory. The relative energies with respect to the reactants as well as the structures of the stationary points are shown. Blue, red and white spheres represent nitrogen, oxygen, and hydrogen atoms. b, c Rotationally adiabatic, centrifugally corrected long-range interaction potentials for the reaction of the ground states of $o-(\mathbf{b})$ and $p-(\mathbf{c}) \mathrm{H}_{2} \mathrm{O}$ with $\mathrm{N}_{2} \mathrm{H}^{+}$for different values of the total angular momentum quantum number $J . \ln \mathbf{b}$, the dashed (solid) lines correspond to the $|\Omega|=0$ (1) components of the ortho-ground state. The grey-shaded areas show an estimate of the uncertainty in the experimental collision energy $E_{\text {col }}$ indicated by the black horizontal line

rate constants were determined to be $k_{o}^{\prime}=1.4(1) \times 10^{-3} \mathrm{~s}^{-1}$ and $k_{p}^{\prime}=1.8(1) \times 10^{-3} \mathrm{~s}^{-1}$ yielding a relative difference $r=23(9) \%$ between the reactivities of the para- and ortho-isomer.

Absolute bimolecular reaction-rate constants $k_{o / p}$ were calculated from $k_{o / p}=k_{o / p}^{\prime} / n_{\mathrm{H}_{2} \mathrm{O}, \mathrm{II}}$, where the total time-averaged beam density at the reference position II, $n_{\mathrm{H}_{2} \mathrm{O}, \mathrm{II}}=3.0(5) \times 10^{5} \mathrm{~cm}^{-3}$, was estimated according to the procedures described in Supplementary Note 5. Using this information, the absolute reaction rates obtained from this experiment are $k_{o}=4.8(9) \times 10^{-9} \mathrm{~cm}^{3} \mathrm{~s}^{-1}$ for ortho-water and $k_{p}=6(1) \times 10^{-9} \mathrm{~cm}^{3} \mathrm{~s}^{-1}$ for para-water, respectively.

\section{Discussion}

To understand the reason for the different reactivities of paraand ortho-water in the present case, ab initio calculations of the energy profile of the reaction were performed (Methods and Supplementary Note 6). As can be seen in Fig. 4a, the reaction was found to be barrierless and to proceed via the formation of an intermediate complex in which one hydrogen atom is shared between the nitrogen and water moieties. No transition state could be located within $\mathrm{O}-\mathrm{H}^{+}$distances ranging from 100 to 260 pm (see Supplementary Fig. 5). The energy of the products was found to be about $2 \mathrm{eV}$ lower than the one of the reactants. This situation suggests that the kinetics can be modelled within the framework of a rotationally adiabatic quantum capture theory for barrierless ion-molecule reactions ${ }^{30,31}$. According to this approach, the reaction rates are entirely dominated by the properties of the long-range interaction potential of the reactants and centrifugal effects. All collisions up to a maximum total angular momentum $J_{\max }$ for which the relative kinetic energy exceeds the height of the centrifugal barrier lead to a successful reactive encounter (Supplementary Note 6).

For the present case, the relevant terms in the long-range interaction potential are the charge-induced dipole and chargepermanent dipole interactions. In the current experiments, both nuclear-spin isomers were cooled down to their relevant rotational ground states. The anisotropic nature of the ion-dipole interaction implies that it is sensitive to the rotational quantum state of the neutral molecule. Figure $4 \mathrm{~b}, \mathrm{c}$ shows rotationally adiabatic, centrifugally corrected interaction potentials for collisions of $\mathrm{N}_{2} \mathrm{H}^{+}$with $o$ - and $p-\mathrm{H}_{2} \mathrm{O}$, respectively, as a function of the total angular momentum quantum number $J$. For the case of vanishing total angular momentum $(J=0)$, one can see that the potential-energy curves for the ortho-species are less steep than the one of the para-isomer, reflecting the stronger rotational averaging of the ion-dipole interaction in the ground state of $o$ $\mathrm{H}_{2} \mathrm{O}$. For the $\left.j_{K_{a} K_{c}}|\Omega|\right\rangle=\left|0_{00} 0\right\rangle$ ground state of the para-species, one obtains a maximum collisional angular-momentum $J_{\max }=$ 166 at the experimental collision energy $E_{\mathrm{col}}=0.019 \mathrm{eV}$. Here, $\Omega$ is the quantum number of the projection of $\vec{j}$ on the distance vector between the ion and the neutral molecule in a body-fixed coordinate system describing the collision ${ }^{30,31}$. This value can be compared to $J_{\max }=151$ and $J_{\max }=146$ for the $\left|1_{00} 0\right\rangle$ and $\left|1_{01} 1\right\rangle$ states of the ortho-isomer, respectively.

The quantum capture model predicts a reaction-rate constant of $k_{p}^{\mathrm{AC}}=5(1) \times 10^{-9} \mathrm{~cm}^{3} \mathrm{~s}^{-1}$ for para-water compared to $k_{o}^{\mathrm{AC}}=$ $4.0(9) \times 10^{-9} \mathrm{~cm}^{3} \mathrm{~s}^{-1}$ for ortho-water. The value for the orthospecies was obtained by summing over the contributions of all $\Omega$ components to the reaction cross section (Supplementary Note 6) assuming that the original preparation of $M$ states of $o-\mathrm{H}_{2} \mathrm{O}$ in the deflector was scrambled during the transit of the molecules 
through the RF fields in the ion trap. In a classical interpretation of this result, the higher maximum angular momentum obtained for the para-isomer implies a larger impact parameter and, therefore, a higher reaction rate. The theoretical predictions agree with the measured values within their experimental uncertainties. The relative difference of the theoretical reaction-rate constants was calculated to be $r=24(5) \%$ which can be compared with the experimental value of $r=23(9) \%$. The errors in the calculated rate constants arise from the uncertainty in the collision energy due to the experimental velocity distribution of the molecular beam and the micromotion of the ions in the large Coulomb crystals $^{20}$ (Supplementary Note 7).

In conclusion, we have studied chemical reactions of the spatially separated ground states of para- and ortho-water with trapped diazenylium ions. We found a $23(9) \%$ higher reactivity for the para nuclear-spin isomer which we attribute to the smaller degree of rotational averaging of the ion-dipole long-range interaction compared to the ortho-species. The observed difference in reactivities is thus a rotational effect which is induced by the nuclear-spin symmetry via the generalized Pauli principle. The present results highlight the interplay between nuclear-spin and rotational symmetry and its ramifications on chemical reactivity. They also provide an illustration of the effects of exchange symmetry on chemical processes which may be put in context with, e.g., its manifestations in the dynamics of ultracold collisions ${ }^{32}$. Measurements such as the ones presented here fill a gap in experimental investigations of ion-neutral reactions with control over the quantum states of the reactants. Indeed, to our knowledge the present study is the first in which rotationally state-selected polyatomic neutral molecules have been reacted with ions. The methods employed here are applicable to studies of a broad range of ion-neutral processes. They enable a quantitative understanding of how different molecular quantum states and, as demonstrated in our earlier study ${ }^{22}$, molecular conformations, influence chemical reactivity.

\section{Methods \\ REMPI spectra. $(2+1)$-resonance-enhanced multiphoton-ionization (REMPI) spectra of $\mathrm{H}_{2} \mathrm{O}$ were acquired using the output of a frequency-doubled dye laser pumped by the $3 \mathrm{rd}$ harmonic $(355 \mathrm{~nm})$ of a Nd:YAG laser. The resulting laser pulses at $248 \mathrm{~nm}$ with an energy of about $1.5 \mathrm{~mJ}$ were focused into the molecular beam using a lens with a focal length of $30 \mathrm{~cm}$. Details on the procedure for the} analysis of the REMPI spectra are given in the Supplementary Note 1.

Femtosecond-laser ionization and ion preparation. Strong-field ionization of $\mathrm{H}_{2} \mathrm{O}$ molecules was performed with pulses from a Ti:Sapphire femtosecond laser (CPA 2110, Clark-MXR, Inc.) at a wavelength of $775 \mathrm{~nm}$ and pulse duration of 150 fs focused down to a beam diameter of $\approx 50 \mu \mathrm{m}$. Similarly, Ca atoms as well as $\mathrm{N}_{2}$ molecules (partial pressure $4.0 \times 10^{-9} \mathrm{mbar}$ ) were ionized using the fs laser before loading into the ion trap in a standardized procedure generating crystals of a constant size and composition as verified by TOF-MS. The $\mathrm{N}_{2}^{+}$ions were reacted with $\mathrm{H}_{2}$ gas leaked into the ultrahigh-vacuum chamber for $30 \mathrm{~s}$ at a partial pressure of $p=2 \times 10^{-9}$ mbar to yield $\mathrm{N}_{2} \mathrm{H}^{+}$ions.

Ion trapping and mass analysis. The ions were trapped in a radio frequency (RF) linear-quadrupole ion trap (LQT) operated at a peak-to-peak RF voltage $V_{\mathrm{RF}, \mathrm{pp}}=$ $800 \mathrm{~V}$ and frequency $\Omega_{\mathrm{RF}}=2 \pi \times 3.304 \mathrm{MHz}$. Doppler laser cooling of $\mathrm{Ca}^{+}$was achieved using two laser beams at $397 \mathrm{~nm}$ and $866 \mathrm{~nm}$ generated by frequencystabilized external-cavity diode lasers ${ }^{20}$. Laser and sympathetic cooling as well as Coulomb crystallization of the ions were monitored by imaging the laser-cooling fluorescence of the $\mathrm{Ca}^{+}$ions onto a camera coupled to a microscope (see sample image inset in Fig. 1). The LQT was connected to a TOF-MS orthogonal to the molecular-beam propagation axis for the mass and quantitative analysis of reactant and product ions 27 .

Molecular beam and Stark deflector. The molecular beam was generated from water vapor at room temperature and seeded in argon carrier gas at 3 bar. The gas mixture was pulsed through an Amsterdam cantilever piezo valve (ACPV2, $150 \mu \mathrm{m}$ nozzle) at a repetition rate of $200 \mathrm{~Hz}$ and a pulse width of $30 \mu \mathrm{s}$. The velocity of the resulting molecular beam was measured to be $575(65) \mathrm{m} / \mathrm{s}$. The electrostatic deflector consists of a pair of $15.4 \mathrm{~cm}$ long electrodes separated by $1.4 \mathrm{~mm}{ }^{23}$.
Theory. Effective dipole moments and Stark energy curves of individual rotationa states of water were calculated for input into Monte-Carlo trajectory simulations using the CMIstark software package ${ }^{33}$. Details of the numerical procedures are reported in the Supplementary Note 1. Ab initio calculations of the potential energy surface of the title reaction were performed using the Gaussian 09 suite of $\operatorname{codes}^{34}$. Geometry optimizations of stationary points were carried out at the CCSD/aug-cc-pVTZ level of theory.

Code availability. The custom computer codes used for calculating Stark curves, for performing Monte Carlo trajectory simulations and for calculating adiabaticcapture rate constants are available from the corresponding author upon request.

Data availability. The data that support the findings of this study are available from the corresponding author upon request.

Received: 26 March 2018 Accepted: 27 April 2018

Published online: 29 May 2018

\section{References}

1. Bunker, P. R. \& Jensen, P. Molecular Symmetry and Spectroscopy. 2nd edn, (NRC Research Press, Ottawa, 1998).

2. Kanamori, H., Dehghani, Z. T., Mizoguchi, A. \& Endo, Y. Detection of microwave transitions between ortho and para states in a free isolated molecule. Phys. Rev. Lett. 119, 173401 (2017).

3. Quack, M. Detailed symmetry selection rules for reactive collisions. Mol. Phys. 34, 477 (1977).

4. Sun, Z.-D., Takagi, K. \& Matsushima, F. Separation and conversion dynamics of four nuclear spin isomers of ethylene. Science 310, 1938 (2005).

5. Sun, Z.-D., Ge, M. \& Zheng, Y. Separation and conversion dynamics of nuclear-spin isomers of gaseous methanol. Nat. Commun. 6, 6877 (2015).

6. Tikhonov, V. I. \& Volkov, A. A. Separation of water into its ortho and para isomers. Science 296, 2363 (2002).

7. Manca Tanner, C., Quack, M. \& Schmidiger, D. Nuclear spin symmetry conservation and relaxation in water $\left({ }^{1} \mathrm{H}_{2}{ }^{16} \mathrm{O}\right)$ studied by cavity ring-down (CRD) spectroscopy of supersonic jets. J. Phys. Chem. A 117, 10105 (2013).

8. Georges, R. et al. Nuclear spin symmetry conservation in ${ }^{1} \mathrm{H}_{2}{ }^{16} \mathrm{O}$ investigated by direct absorption FTIR spectroscopy of water vapor cooled down in a supersonic expansion. J. Phys. Chem. A 121, 7455 (2017).

9. Horke, D. A., Chang, Y.-P., Dlugolecki, K. \& Küpper, J. Separating para and ortho water. Angew. Chem. Int. Ed. 53, 11965 (2014).

10. Guettler, R. D., Jones, G. C. Jr., Posey, L. A. \& Zare, R. N. Partial control of an ion-molecule reaction by selection of the internal motion of the polyatomic reagent ion. Science 266, 259 (1994).

11. Crim, F. F. Chemical dynamics of vibrationally excited molecules: controlling reactions in gases and on surfaces. Proc. Natl Acad. Sci. 105, 12647 (2008).

12. Liu, K. Vibrational control of bimolecular reactions with methane by mode, bond, and stereo selectivity. Ann. Rev. Phys. Chem. 67, 91 (2016).

13. Smith, I. W. M. Reactions at very low temperatures: gas kinetics at a new frontier. Angew. Chem. Int. Ed. 45, 2842 (2006).

14. Chang, Y.-P., Horke, D. A., Trippel, S. \& Küpper, J. Spatially-controlled complex molecules and their applications. Int. Rev. Phys. Chem. 34, 557 (2015).

15. Hauser, D. et al. Rotational state-changing cold collisions of hydroxyl ions with helium. Nat. Phys. 11, 467 (2015).

16. Shagam, Y. et al. Molecular hydrogen interacts more strongly when rotationally excited at low temperatures leading to faster reactions. Nat. Chem. 7, 921 (2015).

17. Perreault, W., Mukherjee, N. \& Zare, R. N. Quantum control of molecular collisions at 1 kelvin. Science 358, 356 (2017).

18. Gerlich, D. et al. State specific stabilization of $\mathrm{H}^{+}+\mathrm{H}_{2}(\mathrm{j})$ collision complexes. J. Chem. Phys. A 117, 10068 (2013).

19. Kravchuk, T. et al. A magnetically focused molecular beam of ortho-water. Science 331, 319 (2011)

20. Willitsch, S. Coulomb-crystallised molecular ions in traps: methods, applications, prospects. Int. Rev. Phys. Chem. 31, 175-199 (2012).

21. Willitsch, S. Chemistry with Controlled Ions, Adv. Chem. Phys. 162, 307 (2017)

22. Chang, Y.-P. et al. Specific chemical reactivities of spatially separated 3aminophenol conformers with cold $\mathrm{Ca}^{+}$ions. Science 342, 98 (2013).

23. Rösch, D., Willitsch, S., Chang, Y.-P. \& Küpper, J. Chemical reactions of conformationally selected 3 -aminophenol molecules in a beam with Coulombcrystallized $\mathrm{Ca}^{+}$ions. J. Chem. Phys. 140, 124202 (2014).

24. Turner, B. E. A new interstellar line with quadrupole hyperfine splitting. Astrophys. J. 193, L83 (1974). 
25. Caselli, P., Benson, P. J., Myers, P. C. \& Tafalla, M. $\mathrm{N}_{2} \mathrm{H}^{+}(1-0)$ maps of dense cloud cores. Astrophys. J. 572, 238 (2002).

26. Bergin, E. A., Alves, J., Huard, T. \& Lada, C. J. $\mathrm{N}_{2} \mathrm{H}^{+}$and $\mathrm{C}^{18} \mathrm{O}$ depletion in a cold dark cloud. Astrophys. J. Lett. 570, L101 (2002).

27. Rösch, D., Gao, H., Kilaj, A. \& Willitsch, S. Design and characterization of a linear quadrupole ion trap for high-resolution Coulomb-crystal time-of-flight mass spectrometry. EPJ Tech. Instrum. 3, 5 (2016).

28. Yang, C.-H., Sarma, G., ter Meulen, J. J., Parker, D. H. \& Western, C. M. REMPI spectroscopy and predissociation of the $\tilde{C}^{1} B_{1}(v=0)$ rotational levels of $\mathrm{H}_{2} \mathrm{O}, \mathrm{HOD}$ and $\mathrm{D}_{2} \mathrm{O}$. Phys. Chem. Chem. Phys. 12, 13983 (2010).

29. Teschmit, N. et al. Characterizing and optimizing a laser-desorption molecular beam source. J. Chem. Phys. 147, 144204 (2017).

30. Clary, D. Rate constants for the reactions of ions with dipolar polyatomic molecules. J. Chem. Soc., Faraday Trans. 2 83, 139-148 (1987).

31. Stoecklin, T., Clary, D. C. \& Palma, A. Rate constant calculations for ionsymmetric top and ion-asymmetric top reactions. J. Chem. Soc. Faraday Trans. 88, 901 (1992).

32. Ospelkaus, S. et al. Quantum-state controlled chemical reactions of ultracold potassium-rubidium molecules. Science 327, 853 (2010).

33. Chang, Y.-P., Filsinger, F., Sartakov, B. G. \& Küpper, J. CMIstark: Python package for the stark-effect calculation and symmetry classification of linear, symmetric and asymmetric top wavefunctions in dc electric fields. Comp. Phys. Comm. 185, 339 (2014).

34. Frisch, M. J. et al. Gaussian 09 Revision E.01. (Gaussian Inc., Wallingford CT, 2009).

\section{Acknowledgements}

We thank Philipp Knöpfel, Grischa Martin, and Georg Holderried for technical support. Dominique Ostermayer and Jolijn Onvlee are acknowledged for their assistance with the experiments and simulations, respectively. This work is supported by the Swiss National Science Foundation under grant nr. BSCGI0_157874.

\section{Author contributions}

D.R. designed and built the apparatus with help from H.G. A.K. and H.G performed the experiments. A.K analysed the data, performed theoretical calculations and simulated spatial beam profiles. D.R. wrote the computer code for Monte-Carlo trajectory simulations and capture-theory calculations. U.R. performed the quantum-chemical calculations. A.K., H.G., J.K., and S.W. wrote the manuscript. S.W. and J.K. conceived and supervised the project. All authors have read and approved the final manuscript.

\section{Additional information}

Supplementary Information accompanies this paper at https://doi.org/10.1038/s41467 018-04483-3.

Competing interests: The authors declare no competing interests.

Reprints and permission information is available online at http://npg.nature.com/ reprintsandpermissions/

Publisher's note: Springer Nature remains neutral with regard to jurisdictional claims in published maps and institutional affiliations.

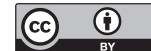

Open Access This article is licensed under a Creative Commons Attribution 4.0 International License, which permits use, sharing, adaptation, distribution and reproduction in any medium or format, as long as you give appropriate credit to the original author(s) and the source, provide a link to the Creative Commons license, and indicate if changes were made. The images or other third party material in this article are included in the article's Creative Commons license, unless indicated otherwise in a credit line to the material. If material is not included in the article's Creative Commons license and your intended use is not permitted by statutory regulation or exceeds the permitted use, you will need to obtain permission directly from the copyright holder. To view a copy of this license, visit http://creativecommons.org/ licenses/by/4.0/.

(C) The Author(s) 2018 